\title{
"CONVEX" CHARACTERIZATION OF LINEARLY CONVEX DOMAINS
}

\author{
NIKOLAI NIKOLOV and PASCAL J. THOMAS*
}

\begin{abstract}
We prove that a $C^{1,1}$-smooth bounded domain $D$ in $C^{n}$ is linearly convex if and only if the convex hull of any two discs in $D$ with common center lies in $D$.
\end{abstract}

\section{Statements}

Recall that an open set $D$ in $\mathrm{C}^{n}$ is called (cf. [1], [3]):

- C-convex if any non-empty intersection with a complex line is connected and simply connected;

- linearly convex if its complement in $\mathrm{C}^{n}$ is a union of affine complex hyperplanes;

- weakly linearly convex if for any $a \in \partial D$ there exists an affine complex hyperplane through $a$ which does not intersect $D$.

Note that the following implications hold:

$$
\text { C-convexity } \Rightarrow \text { linear convexity } \Rightarrow \text { weak linear convexity. }
$$

Moreover, these three notions coincide in the case of bounded domains with $C^{1}$-smooth boundary (cf. [1], [3]).

Let now $D$ be an open set in $C^{n}, z \in D$ and $X \in C^{n}$. Denote by $d_{D}(z, X)$ the distance from $z$ to $\partial D$ in the complex direction $X$ (possibly $d_{D}(z, X)=\infty$ ):

$$
d_{D}(z, X)=\sup \{r>0: z+\lambda X \in D \text { if }|\lambda|<r\} .
$$

Note that the following three properties are equivalent:

- $1 / d_{D}(z, \cdot)$ is a convex function;

* This paper was written during the stay of the second-named author at the Institute of Mathematics and Informatics of the Bulgarian Academy of Sciences (September 2010) supported by a CNRS-BAS programme "Convention d'échanges" No. 23811.

Received 19 October 2010, in final form 19 August 2011. 
- the maximal circular open subset $D_{z}$ of $D$ w.r.t. $z$ is convex $\left(1 / d_{D}(z, \cdot)\right.$ is the Minkowski function of $D_{z}-z$ );

- $D$ contains the convex hull of the union of any two (complex affine) discs in $D$ with center $z$.

By [7] (see also [6]), any weakly linearly convex open set has these properties. To see this directly for the third property, let us compute the linearly convex hull of a union of two discs, which coincides with its convex hull.

By using linear transformations, we may reduce ourselves to the case of $K:=(\overline{\mathrm{D}} \times\{0\}) \cup(\{0\} \times \overline{\mathrm{D}})$ in $\mathrm{C}^{2}$. Then, if we identify a complex hyperplane not passing through 0 with the coefficients $\left(a_{1}, a_{2}\right)$ of its representation as $\left\{a_{1} z_{1}+a_{2} z_{2}=1\right\}$, the (polar) set of all hyperplanes not meeting $K$ is $K^{*}=\mathrm{D}^{2}$. Then (see [1]) the linearly convex hull of $K$ is given by

$$
\left(K^{*}\right)^{*}=\left\{z:\left|z_{1}\right|+\left|z_{2}\right| \leq 1\right\}
$$

which coincides with the convex hull of $K$. (It is also the hull of $K$ with respect the family of linear-fractional functions [1], since those are constant on complex hyperplanes). Therefore any weakly linearly convex open set must contain the convex hull of the union of two affine discs contained in the domain and intersecting at their common center.

Our aim is to show that the converse is also true in the case of $C^{1,1}$-smooth bounded domains. We do not know if this regularity can be weakened. Nonsmooth linearly convex domains can be quite different (they can fail to be C-convex), and Aizenberg's question is still open: can any C- convex domain be exhausted by smooth C-convex domains?

Proposition 1. Let $D$ be a $C^{1,1}$-smooth bounded domain in $C^{n}$ and let $U$ be a neighborhood of $\partial D$. If $D$ contains the convex hull of any two discs in $D \cap U$ with common center, then $D$ is linearly convex.

This can be considered as an analogue of the characterization of convex domains by line segments, or of pseudoconvex domains by Hartogs figures.

More precisely, it follows from the proof of Lemma 4 that a bounded $C^{1,1}$ smooth domain $D$ is not linearly convex if and only if there are $c \in \partial D$ and a line segment $[a, b]$ in the complex tangent hyperplane at $c$ such that $c$ is its midpoint and $[a, b] \backslash\{c\} \subset D$. This is analogous to the situation for real convexity.

Proposition 2. Let $U$ be a neighborhood of the boundary of a domain $D$ in $\mathrm{R}^{n}$ such that if $D \cap U$ contains two sides of a triangle, then it contains the midpoint of the third side. Then $D$ is convex. 
Sketch of the PRoof. Assume that $D$ is not convex. Then there is a boundary point $c$ which is the midpoint of a segment $[a, b]$ with $[a, b] \backslash\{c\} \subset D$ (cf. [3, Theorem 2.1.27]). To get a contradiction, it is enough to find $d \in D$ near $c$ such that $[a, d] \subset D$ and $[b, d] \subset D$. This follows by [3, Theorem 2.1.27], since we may touch $D$ at $z$ from inside by a smooth domain not convex at $c$.

\section{Proofs}

Proof of Proposition 1. First we need a slight modification of $[4$, Theorem 1.4].

Proposition 3. Suppose that D has a $C^{1,1}$-smooth boundary, with defining function $\rho$. If for almost every $p \in \partial D$

$$
\liminf _{T^{\complement}(p) \ni \zeta \rightarrow p} \frac{\rho(\zeta)}{|\zeta-p|^{2}} \geq 0
$$

where $T^{\mathrm{C}}(p)$ denotes the largest complex affine subspace passing through $p$ and contained in the real affine tangent space to $\partial D$, then $D$ is linearly convex.

Notice that [4, Theorem 1.4] has the same conclusion with slightly different hypotheses: it demands a little less boundary regularity of $D$, but assumes the inequality (1) everywhere instead of almost everywhere. When $D$ has a $C^{2}$ smooth boundary, the second partials are defined and continuous everywhere, and so (1) holds everywhere and there is nothing more to prove.

In Section 3, we shall recall the steps of Hörmander's proof and give the small modifications needed to adapt it to our context.

Assume to get a contradiction that $D$ is not linearly convex. By the Implicit Function Theorem, we can choose local coordinates such that the boundary of $D$ can be written locally as a graph. By Rademacher's theorem about the differentiability almost everywhere of Lipschitz functions, applied to the first partial derivatives of the function defining the graph in each coordinate patch, we may find a point $p \in \partial D$ such that $\rho$ is twice differentiable at $p$ and

$$
\liminf _{T^{c}(p) \ni \zeta \rightarrow p} \frac{\rho(\zeta)}{|\zeta-p|^{2}}<0 .
$$

From now on, we choose such a $p$.

It is easy to show that the property we are studying can be tested on twodimensional subspaces, so henceforth we assume that $\Omega \subset \mathrm{C}^{2}$.

Lemma 4. Under the above hypotheses, there exist $r>0, c \geq 1$ and coordinates $(z, w)$ obtained by a complex affine transformation from the original coordinates such that $(z(p), w(p))=(0,0)$ and

$$
\Omega \cap \mathrm{B}_{2}(0, r) \supset E:=\left\{(z, w) \in \mathrm{C}^{2}: \rho_{c}(z, w)<0\right\} \cap \mathrm{B}_{2}(0, r),
$$


where $\rho_{c}(z, w)=\operatorname{Re} z-(\operatorname{Re} w)^{2}+c|z|^{2}+c(\operatorname{Im} w)^{2}$.

Proof. It will be enough to majorize $\rho$ by $\rho_{c}$ for some $c>0$ when $(z, w)$ is close enough to $p$.

First take coordinates $\left(z_{1}, w_{1}\right)$ such that $\left(z_{1}(p), w_{1}(p)\right)=(0,0)$ and the real gradient $\nabla \rho(0,0)=(1,0)$. Then by Taylor's formula,

$$
\begin{aligned}
\rho\left(z_{1}, w_{1}\right)= & \operatorname{Re} z_{1}+\operatorname{Re}\left(a_{11} z_{1}^{2}+a_{12} z_{1} w_{1}+a_{22} w_{1}^{2}\right) \\
& +b_{11}\left|z_{1}\right|^{2}+\operatorname{Re}\left(b_{12} z_{1} \bar{w}_{1}\right)+b_{22}\left|w_{1}\right|^{2}+o\left(\left|z_{1}\right|^{2}+\left|w_{1}\right|^{2}\right),
\end{aligned}
$$

where the coefficients $a_{i j}, b_{i j}$ are deduced from the second order partial derivatives of $\rho$ at $(0,0)$ in the usual way.

Here $T^{\complement}(p)=\{(0, w), w \in \mathrm{C}\}$, and

$$
\frac{\rho\left(0, w_{1}\right)}{\left|\left(0, w_{1}\right)-p\right|^{2}}=\operatorname{Re}\left(a_{22} \frac{w_{1}^{2}}{\left|w_{1}\right|^{2}}\right)+b_{22}+o(1),
$$

and the liminf in (2) is exactly $-\left|a_{22}\right|+b_{22}=:-\ell$.

We rotate the $w_{1}$ coordinate so that $a_{22}=-\left|a_{22}\right|$, thus

$$
\rho\left(0, w_{1}\right)=-\ell\left(\operatorname{Re} w_{1}\right)^{2}+\left(2 b_{22}+\ell\right)\left(\operatorname{Im} w_{1}\right)^{2}+o\left(\left|w_{1}\right|^{2}\right) .
$$

To estimate the other terms,

$$
\left|a_{12} z_{1} w_{1}+b_{12} z_{1} \bar{w}_{1}\right| \leq c_{1}\left|z_{1} w_{1}\right| \leq \frac{1}{2} c_{1}\left(\varepsilon\left|w_{1}\right|^{2}+\frac{1}{\varepsilon}\left|z_{1}\right|^{2}\right) ;
$$

we choose $\varepsilon$ so that $c_{1} \varepsilon \leq \ell, c_{2}:=\left|a_{11}\right|+\left|b_{11}\right|$, so

$$
\begin{aligned}
\rho\left(z_{1}, w_{1}\right)< & \operatorname{Re} z_{1}+\left(c_{2}+\frac{c_{1}}{2 \varepsilon}\right)\left|z_{1}\right|^{2}-\frac{\ell}{2}\left(\operatorname{Re} w_{1}\right)^{2}+\left(\frac{\varepsilon c_{1}}{2}+2 b_{22}+\ell\right)\left(\operatorname{Im} w_{1}\right)^{2} \\
& +o\left(\left|z_{1}\right|^{2}+\left|w_{1}\right|^{2}\right) \leq \operatorname{Re} z_{1}+c_{3}\left|z_{1}\right|^{2}-\frac{\ell}{3}\left(\operatorname{Re} w_{1}\right)^{2}+c_{4}\left(\operatorname{Im} w_{1}\right)^{2}
\end{aligned}
$$

for $\left(z_{1}, w_{1}\right)$ small enough.

Taking $z=z_{1}, w=\sqrt{\frac{\ell}{3}} w_{1}$, we have the required form.

Further, choose $r>0$ such that $\mathrm{B}_{2}(0, r) \subset U$ and suppose that we have two disks in $E$ of the form

$$
\begin{aligned}
& D_{1}=\{(-\delta(1-\zeta), \delta \zeta / \mu),|\zeta| \leq 1\}, \\
& D_{2}=\{(-\delta(1+\zeta), \delta \zeta / \mu),|\zeta| \leq 1\},
\end{aligned}
$$


where $\mu=\sqrt{2 c \delta}$. Then by taking the midpoint of $(0, \delta / \mu)\left(\zeta=1\right.$ for $\left.D_{1}\right)$ and $(0,-\delta / \mu)\left(\zeta=-1\right.$ for $\left.D_{2}\right)$ we find $(0,0)$, which is a contradiction, proving Proposition 1.

One can see by changing $\zeta$ into $-\zeta$ that it is enough to check that $D_{1} \subset E$. It is clear that $D_{1} \subset \mathrm{B}(0, r)$ for any small $\delta>0$. It remains to show that $\left.\rho_{c}\right|_{D_{1}}<0$ on $D_{1}$. Since $c \geq 1$, this restriction is subharmonic and so it suffices to prove the inequality for $\zeta=e^{i \theta}$.

Dividing through by $\delta$, we have to verify

$$
-1+\cos \theta-\frac{1}{2 c} \cos ^{2} \theta+\frac{1}{2} \sin ^{2} \theta+2 c \delta(1-\cos \theta)<0, \quad \theta \in \mathbf{R},
$$

equivalently

$$
-1+4 c \delta+2(1-2 c \delta) x-\left(1+\frac{1}{c}\right) x^{2}<0, \quad-1 \leq x \leq 1 .
$$

Computing the (reduced) discriminant of this quadratic polynomial yields

$$
(1-2 c \delta)^{2}-\left(1+\frac{1}{c}\right)(1-4 c \delta)=4 c^{2} \delta^{2}+4 \delta-\frac{1}{c}<0
$$

for $\delta>0$ small enough.

The proof of Proposition 1 is completed.

\section{Appendix}

Proof of Proposition 3. First, the hypothesis and conclusion of [4, Theorem 1.4] are restated in terms of the function

$$
h(z):=\inf _{w \in \partial D}|z-w|^{2}, \quad z \in D .
$$

For any $\zeta \in \partial D$ at which this infimum is attained, $z$ belongs to the normal to $\partial D$ at $\zeta$. For any $z$ for which the infimum is attained at a unique point $\zeta \in \partial D$, we denote $\pi(z)=\zeta$ this nearest point. Then $h$ is differentiable at any point $z$ where $\pi(z)$ is well defined.

The property that $\partial D$ admits a neighborhood in which the nearest point in uniquely defined is called "positive reach" in [2]. When $\partial D$ is $\mathscr{C}^{1,1}$ smooth (i.e., $D$ admits a $\mathscr{C}^{1,1}$-smooth defining function with non vanishing gradient near the boundary), it is of positive reach, as follows for instance from the remark at the beginning of [2, Section 4], "the class of sets with positive reach is closed under bi-Lipschitzian maps with Lipschitzian differentials" (or see the note added in proof at the end of [5]). 
It is proved in [5] that when $\partial D$ has positive reach and is $\mathscr{C}^{1}$, then $h \in \mathscr{C}^{1}$ and its first partial derivatives are given explicitly in terms of the first partial derivatives of the defining function in an appropriate coordinate system, see equations (3) and (4) on [5, p. 118]. Since the $\mathscr{C}^{1,1}$ property does not depend on the coordinate system, it follows from the formulae that if $\rho$ has Lipschitz continuous first partial derivatives, then so does $h$, i.e., $h \in \mathscr{C}^{1,1}$. Note that for any $\varepsilon>0$ there exist domains with $C^{2-\varepsilon}$-smooth boundary which do not have positive reach, and where $h$ fails to be differentiable in a neighborhood of the boundary, so $\mathscr{C}^{1,1}$ smoothness is a kind of minimal hypothesis we have to demand using these methods.

It is also noted in a remark after the statement of [4, Theorem 1.4] that when $\partial D$ is $\mathscr{C}^{1,1}$-smooth, the regularity hypotheses of the theorem, i.e., the interior ball condition (which implies positive reach) and condition [4, (1.5)], are in particular fulfilled.

[4, Proposition 1.5] shows that $D$ is linearly convex if and only if

(3) $h(w) \leq h(z)+2 \operatorname{Re}\left\langle w-z, h_{z}^{\prime}(z)\right\rangle+\left|\left\langle w-z, h_{z}^{\prime}(z)\right\rangle\right|^{2} / h(z), \quad w \in D$,

for every $z$ at which $h$ is differentiable.

Then the proof of [4, Theorem 1.4], given at the end of [4, Section 2], proceeds as follows: given a $\delta>0$ such that $h$ is $\mathscr{C}^{1}$ on $\mathrm{D}_{\delta}:=\left\{h<\delta^{2}\right\}$, choose for each $\zeta \in \partial D$ a ball $B_{\delta, \zeta}$ of radius $\delta / 2$, tangential to $\partial D$ at $\zeta$ (and thus contained in $\mathrm{D}_{\delta}$ ). It is shown that $D$ is linearly convex if (3) holds on each $B_{\delta, \zeta}$. For that it is enough to show that $h$ is quadratically concave, a notion defined by [4, Definition 2.1].

[4, Theorem 2.4] shows that when a positive function $g$ is defined on a ball $B \subset R^{N}$ and its second derivatives are measures, a sufficient condition for it to be quadratically concave is that

$$
\left\langle g^{\prime \prime}(z) v, v\right\rangle \leq \frac{1}{2}|v|^{2}\left|g^{\prime}(z)\right|^{2} / g(z),
$$

for any $z \in B, v \in \mathrm{R}^{N}$.

We need to show that the conclusion of [4, Theorem 2.4] still holds under our new hypotheses.

[4, Proposition 1.6] shows that (1) at $\zeta \in \partial D$ implies

(5) $\limsup _{w \rightarrow 0} \frac{1}{|w|^{2}}\left(h(z+w)-h(z)-2 \operatorname{Re}\left\langle w, h_{z}^{\prime}(z)\right\rangle-\frac{\left|\left\langle w, h_{z}^{\prime}(z)\right\rangle\right|^{2}}{h(z)}\right) \leq 0$ at any point $z$ at which $h$ is differentiable, and such that $\zeta=\pi(z)$. Since $z$ must lie in the normal to $\partial D$ at $\zeta$ and $\partial D \in \mathscr{C}^{1,1}$, the implicit function 
theorem shows that if $N \subset \partial D$ is of (2n-1)-Lebesgue measure zero, then in a neighborhood of $\partial D, \pi^{-1}(N)$ is of $2 n$-Lebesgue measure zero. So our hypothesis implies that (5) holds for almost every $z$ in a neighborhood of the boundary. Applying Taylor's formula, we see that this implies that (4) holds for a.e. $z$ in this neighborhood and any vector $v$. So our hypotheses differ from Hörmander's only in that the second derivatives of our function are measures given by a.e. defined bounded functions, and by requiring a secondorder differential inequality almost everywhere instead of everywhere.

[4, Theorem 2.4] shows that $g$ is quadratically concave by proving that

$$
g(y) \leq g(x)+\left\langle y-x, g^{\prime}(x)\right\rangle+\frac{1}{4}|y-x|^{2} \frac{\left|g^{\prime}(x)\right|^{2}}{g(x)}, \quad x, y \in B,
$$

which is enough by [4, Theorem 2.2].

Assuming that $B$ is the unit ball, if we prove (6) for $g(x)=h_{r}(x):=h(r x)$, and $0<r<1$, we obtain the same inequality for $h=h_{1}$, using the fact that $h \in \mathscr{C}^{1}$ to pass to the limit. So we may now assume that $g \geq \varepsilon_{0}>0$. Then we follow part (c) of the proof of [4, Theorem 2.4]: writing $g_{\varepsilon}(x):=$ $g(x)-\varepsilon\left(|x|^{2}+1\right)$ for small enough $\varepsilon>0$, we have that

$$
\left\langle g_{\varepsilon}^{\prime \prime} t, t\right\rangle \leq\left(\frac{1}{2}\left|g_{\varepsilon}^{\prime}(x)\right|^{2} / g_{\varepsilon}(x)-2 \varepsilon /\left(|x|^{2}+1\right)\right)|t|^{2},
$$

and so a $\mathscr{C}^{\infty}$ regularization of $g_{\varepsilon}$ will satisfy (4) with strict inequality in $\bar{B}$ when $|t|=1$ (since the second order differential inequality is obtained by integrating against the regularizing kernel bounded functions that satisfy the inequality almost everywhere). By parts (a) and (b) of the proof of [4, Theorem 2.4] (which are valid for $\mathscr{C}^{2}$-smooth functions), this regularization will satisfy (6), and letting the regularization tend to $g$, we obtain (6) for $g$.

\section{REFERENCES}

1. Andersson, M., Passare, M., Sigurdsson, R., Complex Convexity and Analytic Functionals, Progr. in Math. 225, Birkhäuser, Basel 2004.

2. Federer, H., Curvature measures, Trans. Amer. Math. Soc. 93 (1959), 418-491.

3. Hörmander, L., Notions of Convexity, Progr. in Math. 127, Birkhäuser, Boston 1994.

4. Hörmander, L., Weak linear convexity and a related notion of concavity, Math. Scand. 102 (2008), 73-100.

5. Krantz, S. G., Parks, H. R., Distance to $\mathscr{C}^{k}$ hypersurfaces, J. Differential Equations 40 (1981), $116-120$.

6. Nikolov, N., Pflug, P., Zwonek, W., An example of a bounded C-convex domain which is not biholomorphic to a convex domain, Math. Scand. 102 (2008), 149-155. 
7. Znamenskiŭ, S. V., Znamenskaya, L. N., Projective convexity in $\mathrm{CP}^{n}$, Siberian Math. J. 38 (1997), 685-698.

INSTITUTE OF MATHEMATICS AND INFORMATICS BULGARIAN ACADEMY OF SCIENCES

113 SOFIA

BULGARIA

E-mail: nik@math.bas.bg
UNIVERSITÉ DE TOULOUSE

UPS, INSA, UT1, UTM

INSTITUT DE MATHÉMATIQUES DE TOULOUSE F-31062 TOULOUSE

FRANCE

E-mail: pthomas@math.univ-toulouse.fr 